BULLETIN Bulletin hispanique

HISPANIQUE Université Michel de Montaigne Bordeaux

110-1 | 2008

Varia

\title{
Milagros Ezquerro, Leerescribir
}

\author{
Isabelle Tauzin
}

\section{OpenEdition}

Journals

Édition électronique

URL : https://journals.openedition.org/bulletinhispanique/711

DOI : 10.4000/bulletinhispanique.711

ISSN : 1775-3821

\section{Éditeur}

Presses universitaires de Bordeaux

\section{Édition imprimée}

Date de publication : 1 juin 2008

Pagination : 393-395

ISBN : 978-2-86781-511-9

ISSN : 0007-4640

Référence électronique

Isabelle Tauzin, « Milagros Ezquerro, Leerescribir», Bulletin hispanique [En ligne], 110-1 | 2008, mis en ligne le 10 février 2014, consulté le 18 septembre 2021. URL : http://journals.openedition.org/ bulletinhispanique/711; DOI : https://doi.org/10.4000/bulletinhispanique.711 
particulièrement utile à cet égard, car comme le dit l'auteur (p. 34) «las canciones casi siempre tiran de otros recuerdos»-, non pas comment "fue el pasado en el pasado ", mais comment " el pasado se continúa o perpetúa en el presente ".

J.-F. BOTREL

Milagros Ezquerro, Leerescribir. - Mexico-Paris, Rilma-ADEHL, 2008, 197 p. - ISBN 978-970-95635-1-1.

La professeure Milagros Ezquerro présente avec Leerescribirl'aboutissement de nombreuses années de recherche, toute une carrière consacrée à l'analyse littéraire et à la théorie de la littérature. Milagros Ezquerro est une éminence de l'américanisme français, fidèle à l'analyse d'un corpus textuel qui ne cesse de s'accroître.

Leerescribirvient de sortir, édité à Mexico, en partenariat avec les universités de Limoges et de la Sorbonne. Il s'agit du $5^{\mathrm{e}}$ titre de la collection Estudios y ensayos qu'animent Milagros Ezquerro et le professeur Eduardo RamosIzquierdo. Leerescribir est un mot-valise, autrement dit un néologisme. Ce qu'entend démontrer Milagros Ezquerro, et qu'annonce le titre de cet essai, c'est que lire et écrire sont deux actes qui se télescopent en permanence. L'un n'a pas de sens sans l'autre; l'un est l'autre.

Dans cette démonstration, Milagros Ezquerro rappelle que le texte littéraire est un système complexe qui fonctionne en relation avec deux autres systèmes tout aussi complexes quoique cette complexité soit imperceptible au lecteur lambda. L'approche de Milagros Ezquerro rappelle celle de la physique nucléaire. Mais sa démonstration n'exige pas de connaître une infinité de théorèmes. Pour les scientifiques de la matière, l'atome est la plus petite partie d'un corps simple ; il est généralement constitué d'un noyau autour duquel gravitent des électrons. Dans Leerescribir, Milagros Ezquerro définit d'une part le producteur du texte, qu'elle appelle le Sujet Alpha et l'observateur du texte, le Sujet Omega. Ces deux Sujets aux identités plurielles gravitent et échangent mutuellement avec l'objet qui est au centre de tout : le texte littéraire.

Le sujet Alpha ne se confond pas avec l'auteur, qui écrit le texte. C'est l'auteur mais aussi tout son environnement, tout ce qui a participé au processus de création, la culture de l'auteur, sa vie privée ou publique, les agents secondaires de la production, qu'il s'agisse des maîtres à penser ou des disciples, de l'éditeur qui impose un titre ou de l'illustrateur. Le concept de Sujet Alpha est donc novateur; il s'efforce d'embrasser la totalité du 
processus de production littéraire ou artistique. En outre, le Sujet Alpha évolue, en fonction notamment de la réception de l'œuvre. Les observations du public interagissent aussi bien sur la lecture que le créateur fait ensuite de ses écrits, que pour sa production future. En guise d'exemples Milagros Ezquerro choisit des situations extrêmes mais, oh combien illustratives! Homère, le poète aveugle dont on ne sait rien, ou encore l'auteur des Versets Sataniques, qui fut victime des pires menaces, et aussi Góngora, le grand poète baroque, exhumé en 1927 par les jeunes poètes qui ont décidé de célébrer le troisième centenaire de sa mort. Dès lors, la lecture de Góngora a été transformée. A Góngora, noyau dur du Sujet Alpha se sont agrégés les électrons libres que furent ces poètes découvreurs de 1927. Les figures universelles, les grands noms de chaque littérature nationale acquièrent ainsi une autre identité au fil du temps, différente du noyau générateur.

Milagros Ezquerro met en lumière l'existence, comme dans un miroir, d'un autre système complexe, le lecteur-récepteur-observateur qu'elle dénomme Sujet Omega. Oméga, car le concept englobera à la fois l'idée d'observation, symbolisée en quelque sorte par la lettre $\mathrm{O}$ : ojo, œil, ouverture et aussi aboutissement connoté par la dernière lettre de l'alphabet grec. Cependant, le Sujet Omega ne se contente pas de déchiffrer; il n'est pas seulement récepteur ou destinataire d'un texte. Lui aussi interprète, déchiffre et se remémore d'autres lectures au fur et à mesure qu'il découvre un texte. Plus le texte est éloigné de sa culture, plus le Sujet Omega doit mobiliser d'énergie pour accéder à cet objet inconnu. Milagros Ezquerro soutient et montre que le texte, tout comme le Sujet Alpha et le Sujet Omega, sont des systèmes qui s'auto-organisent au fur et à mesure de l'écriture et de la lecture.

Le producteur du texte est aussi son premier lecteur et il ne se relit jamais de la même façon. Nous-même lisons de façon différente à chaque lecture, un acte qui n'est pas seulement décodage mais aussi nouvel encodage. La part d'indétermination du texte littéraire en fait la richesse, à la différence de la notice d'un produit pharmaceutique, à sens unique, en quelque sorte, excepté lorsqu' intervient, par exemple, l'intention humoristique.

Chaque texte intègre une sorte de galaxie, le sémiotope et génère aussi cette galaxie. La langue dans laquelle est écrit le texte, le genre littéraire, le rythme, les traits d'identité qui permettent de le relier à d'autres textes, tous ces réseaux constituent un imaginaire lieu de signes, le sémiotope.

Le sémiotope, concept que Milagros Ezquerro compare de façon didactique à la notion aujourd'hui bien connue de biotope, est un ensemble complexe fortement dépendant de l'environnement propre au Sujet Alpha. Ce sont toutes les relations que le texte entretient avec le langage, la littérature, la culture, les traditions, le contexte dans toute sa complexité. Le 
sémiotope est le lieu d'échange, au confluent de deux autres lieux conceptuels, les idiotopes selon la terminologie de Milagros Ezquerro, ces lieux imaginaires propres au producteur et à l'observateur du texte, des lieux infinis qui rassemblent toutes les circonstances de la création et de l'acte de lecture.

La succession d'articles qui constituent Leerescribir permettent de comprendre ces concepts déroutants d'idiotope, sémiotope, Sujet Alpha et Sujet Omega, sans que la référence théorique soit toujours explicite. Le lecteur de Milagros Ezquerro, qui les découvre avec ce livre, se trouve dans la position du Sujet Omega, confronté à l'inconnu, comme à une langue étrangère, mais dont peu à peu il s'imprègne, si bien qu'à la fin de la lecture, il a l'impression de pouvoir lui aussi communiquer désormais dans les mêmes termes.

Telle a été mon expérience à la lecture de cet ouvrage, effrayée d'abord par la terminologie mais ensuite rassurée par l'exposé méthodique et les exemples suggestifs, empruntés à la littérature classique. Leerescribir s'apparente à un pas à pas: chaque essai ou chapitre a la brièveté d'une escale : le lecteur avance en découvrant, puis reprend son souffle avant de continuer la route pour aboutir ensuite à l'exposé pratique : la deuxième partie de l'ouvrage composée d'articles plus longs qui sont autant de lecturécritures de grands textes de la littérature latino-américaine, García Márquez et El coronel no tiene quien le escriba, le Paraguayen Roa Bastos, les Uruguayens Quiroga et Hernández, et puis les Argentins Cortázar, Puig, Ocampo et Noemí Ulla. Auteurs anciens et génération contemporaine, les leçons que donne ici magistralement Milagros Ezquerro, nous invitent à la relecture ou à la découverte de ces textes que nous avons l'occasion de connaitre avec d'autres yeux, en partie déjà sous influence.

Les leçons transmises dans Leerescribir reposent en outre sur un questionnement des principaux ouvrages de la critique littéraire du $\mathrm{XX}^{\mathrm{e}}$ siècle (de Paul Valéry à Wolfgang Iser et Hans Jauss). Ces leçons sont exemplaires d'une forme d'analyse en quête d'une solidarité intrinsèque entre les différents éléments constituants de la littérature. Au terme de notre lecture, l'appréhension initiale a disparu et nous nous apercevons que nous pratiquions en partie la Lecturécriture sans le savoir. Nous la pratiquerons désormais, en connaissance de cause, dotée d'un appareil critique savamment construit.

Isabelle TAUZIN 